Desingh Sivanesan

Uthirapathy Natarajan

Veeramalai Chinnasamy Sathish Gandhi

https://doi.org/10.21278/TOF.42407

ISSN 1333-1124

eISSN 1849-1391

\title{
EXPERIMENTAL AND FINITE ELEMENT STUDIES OF A SOFT ROBOT FINGER MATERIAL - A CONTACT MECHANICS APPROACH
}

\begin{abstract}
Summary
A robot is a machine, especially a programmable one, which is capable of carrying out a complex series of actions automatically, emulating certain human movements and functions. Robotic applications are used worldwide to improve quality and to meet production requirements. In this paper, a study has been made to develop a robot finger material which enables a soft contact and withstands a high temperature. Two materials, the Viton ${ }^{\mathrm{R}}$ and the silicone rubber are investigated. Experimental studies on these two materials are conducted to estimate their tensile strength and hardness. Other parameters such as contact width and percent elongation are also determined. The finite element software "ABAQUS" is used for the determination of the Von-Mises stress, contact pressure and contact width at different depths of indentation. In the robot finger design, the properties of the Viton ${ }^{\mathrm{R}}$ rubber are observed to be superior to those of the silicone rubber material.
\end{abstract}

Key words: $\quad$ robot finger, tensile strength, contact pressure, soft contact, Abaqus.

\section{Introduction}

In industry, robots are used for carrying out multiple actions to help the man in a planned manner. The arm of a robot is used in production for different purposes, such as material handling, machine loading and unloading, welding, painting, and assembly. A significant task of the end-effector is to grip and hold objects which are to be moved from one place to another. Soft contact interaction is important in the robot end-effector design. The end-effector has a number of fingers and joints with various degrees of freedom. These factors provide different grasping abilities, like human finger grasping, for example [1]. The most popular finger gripper is a two or a three finger gripper. A vital aspect that is expected of a robot in object gripping is soft contact interaction. Different contact models are used to analyse the object grasping in order to describe the transmission of force between an object and the finger [2]. For linear elastic models, the first contact theory was developed in [3] and then it was modified in [4]. 


\section{Literature Review}

In 1875, Reuleaux started his work on robotic grasping. The first elastic-fingered robot hand was developed in 1979 [5]. In the design of a robot hand, various developments, from simple devices to most stylish multi-fingered Utah MIT hand, have taken place [6]. In the finger - object contacts of multi-finger grasping, the elastic contact model is studied by various approaches [7]. Rigid body models are inadequate for the dynamic analysis and simulation of processes with multiple frictional contacts. In particular, two severe limitations are demonstrated in this paper: (a) The lack of a suitable model for collisions and impacts; (b) Incompatibility with empirical laws of friction such as Coulomb's law of friction [8]. The theory of elastic membrane was developed by the liquid-filled membrane model for the fingertip bulb. This model is helpful for the illustration of large strain deformations [9]. Communicating voids of the internal layer are sealed and hyper elastic models of the continuous outer skin layer are filled with a viscous fluid [10]. The contact mechanics of soft fingertip is studied by developing a contact model. The model is developed by the finite element method. The results are then experimentally validated [11]. The robot finger in a multi-layer structure model was studied and a simulation structure of the human finger robotics was carried out [12]. A multi-layer structure model was considered and a simple model was developed [13]. A type of a robotic finger that is very similar to the human finger is a thin-layered robotic finger filled with fluids. A hyper-elastic, $V_{i t o n}{ }^{\mathrm{R}}$ fluoroelastomer finger model of a semi-cylindrical type, which is filled with a fluid, is to be designed and various parameters, such as tensile strength, compressive strength, load carrying capacity, and tear strength, are to be estimated to validate the material suitability [14]. A review of soft materials for soft robot manipulation is carried out. Various soft materials, like polymermatrix composites and their fillers, are discussed in terms of their advantages and processing methods [15]. The use of additive manufacturing (AM) for the design of a robot gripper for food handling is presented. The AM actuator and grippers developed as a single part is discussed [16]. For power grasping, a contact model of a robot soft finger is applied. A nonlinear model of the soft finger is analysed and results concerning the contact surface with respect to the contact angle are reported [17]. The design and fabrication process of a fully integrated soft humanoid robotic hand with five fingers that integrate an embedded shape memory alloy (SMA) actuator and a piezoelectric transducer (PZT) flexure sensor are discussed [18]. The contact between a robotic or a prosthetic hand and the external world is realised through the artificial skin. Tactile sensors are used for this study. These sensors are normally embedded in soft, synthetic materials used for protecting the subsurface sensor from damage or for establishing a better hand-to-object contact [19]. For robotic applications, a skin like soft material is developed by blending different grades of silicone at 1:1 volume fraction. Five different grades of pure silicone are also used for making skin-like soft materials. The prepared materials are experimentally investigated. A tensile test is then carried out and it is concluded that the tensile strength of $2.189 \mathrm{MPa}$ is obtained while using the Luperox 101 cross-linked silicone grade 10. This is the material that resembles closely the human skin on the forearm [20]. SILASTIC P-1 Silicone, a hyper elastic material supplied by Dow Corning ${ }^{\circledR}$ is considered for the development of a soft actuator. Uniaxial tensile testing and compression testing according to the ASTM standards are conducted to determine its mechanical properties. The mechanical properties of the selected material, such as ultimate tensile strength, compressive strength, shear modulus, and non-linear material constants are presented and used for the design and analysis of the soft actuator for a further study [21]. The material exhibits softness comparable with that of biological tissue and can sustain very high strain [22]. 


\section{Materials and Methods}

Silicone rubber is considered as a finger material and tensile tests are conducted [16]. Other possible elastic materials which could replace silicone rubber are Viton $^{\mathrm{R}}$ and Kalriz ${ }^{\mathrm{R}}$. Viton $^{\mathrm{R}}$ is economically the most suitable finger material. The silicone and Viton ${ }^{\mathrm{R}}$ rubber specimens are shown in Fig. 1 (a) and (b), respectively.

Various properties, such as hardness, Young's modulus, thermal properties, tensile strength and elongation (at break) of the silicone rubber and the Viton ${ }^{\mathrm{R}}$ fluoro elastomer have been evaluated. Various contact parameters, such as contact width, contact pressure, and equivalent stress are estimated in the simulation software 'ANSYS'.

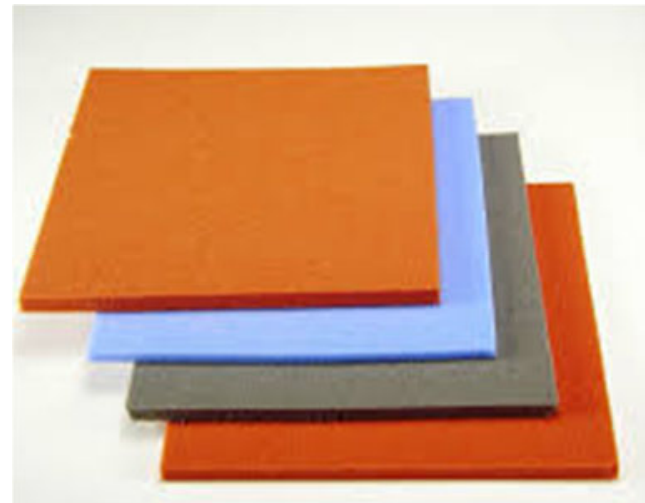

Fig. 1 (a) Silicone rubber

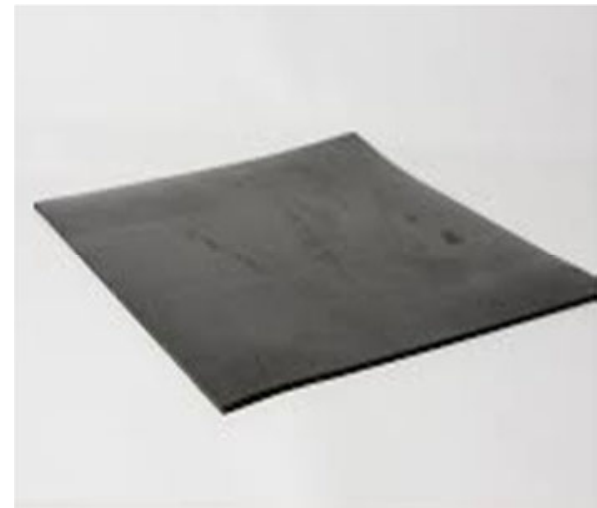

Fig. 1 (b) Viton $^{\mathrm{R}}$ rubber

\subsection{Hardness test}

Hardness is defined as the ability of a material to resist plastic deformation, usually by indentation. Figure 2 shows a hardness tester, Durometer. The hardness values of the Viton ${ }^{\mathrm{R}}$ and silicone rubbers are determined and shown in Table 1.

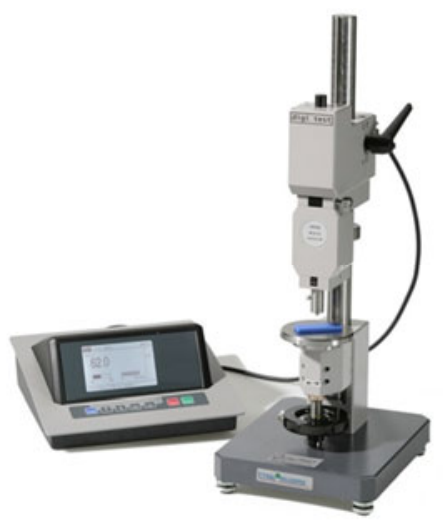

Fig. 2 Durometer - the Shore hardness tester

Table 1 Hardness (S) value

\begin{tabular}{|c|c|}
\hline \multicolumn{2}{|c|}{ Materials } \\
\hline Silicone rubber & Viton $^{\mathrm{R}}$ rubber \\
\hline $65 \mathrm{BHN}$ & $75 \mathrm{BHN}$ \\
\hline
\end{tabular}

\subsection{Young's modulus}

Using the linear elastic indentation hardness, a relation between the ASTM D2240 hardness and Young's modulus for elastomeric materials has been derived in [23] and in [24]. The relationship is shown in equation (1) 
D. Sivanesan, U. Natarajan, V.C. Sathish Gandhi
Experimental and Finite Element Studies of a Soft Robot Finger Material - a Contact Mechanics Approach

$$
E=\frac{0.0981(56+7.62336 S)}{0.137505(254-2.54 S)}
$$

where,

$E$ - Young's modulus in MPa.

$S$ - Hardness.

The values of Young's modulus of the Viton ${ }^{\mathrm{R}}$ and silicone rubbers are calculated from equation (1). The calculated values are shown in Table 2.

Table 2 Young's modulus values

\begin{tabular}{|c|c|}
\hline \multicolumn{2}{|c|}{ Young's modulus in $\mathrm{MPa}$} \\
\hline Silicone rubber & Viton $^{\mathrm{R}}$ rubber \\
\hline 4.43 & 7.05 \\
\hline
\end{tabular}

\subsection{Thermal property}

The thermal property (temperature) of the silicone and Viton $^{\mathrm{R}}$ fluoro elastomer rubbers is measured. Dynamic mechanical analysis is carried out to estimate the heat resistance capacity of the silicone and Viton ${ }^{\mathrm{R}}$ rubbers and the result is shown in Table 3.

Table 3 Thermal Property

\begin{tabular}{|c|c|c|c|}
\hline \multirow{2}{*}{ S. No. } & \multirow{2}{*}{ Properties } & \multicolumn{2}{|c|}{ Type of rubber } \\
\cline { 3 - 4 } & & Silicone & Viton $^{\mathrm{R}}$ \\
\hline 1 & Maximum service temperature $/{ }^{\circ} \mathrm{C}$ & 285 & 312 \\
\hline
\end{tabular}

\subsection{Tensile strength and elongation}

Tensile strength and elongation (at break) are estimated for the silicone and Viton ${ }^{\mathrm{R}}$ fluoro elastomer rubbers and the results are shown in Table 4.

Table 4 Tensile strength and elongation

\begin{tabular}{|c|l|c|c|}
\hline \multirow{2}{*}{ S. No. } & \multirow{2}{*}{ Properties } & \multicolumn{2}{|c|}{ Type of rubber } \\
\cline { 3 - 4 } & & Silicone & Viton $^{\mathrm{R}}$ \\
\hline 1 & Tensile strength $/ \mathrm{N} / \mathrm{mm}^{2}$ & 5.20 & 8.46 \\
\hline 2 & Elongation (at break) & $162.28 \%$ & $203.68 \%$ \\
\hline
\end{tabular}

From Tables 1, 2, 3 and 4, one can note that the properties of the Viton ${ }^{\mathrm{R}}$ rubber are superior to those of the silicone rubber as far as the suitability for the robot finger is concerned. Hence, the Viton ${ }^{\mathrm{R}}$ rubber is considered for further study.

\subsection{Contact width}

Contact width is the dimension measured in the indentation area. A10 mm diameter indenter has made an impression in a flat rubber specimen. Loads of $50 \mathrm{~N}, 100 \mathrm{~N}$, and $150 \mathrm{~N}$ (from the catalogue) are considered for an experiment in the hardness testing machine. The indentation diameters (contact width) are marked and then measured by means of a microscope. Table 5 shows the contact width dimensions estimated in the experiment.

Table 5 Contact width

\begin{tabular}{|c|c|c|c|}
\hline Grasping force / N & 50 & 100 & 150 \\
\hline Contact width / mm & 3.89 & 4.59 & 5.25 \\
\hline Depth of indentation / mm & 4 & 7 & 9.5 \\
\hline
\end{tabular}




\subsection{Finite element analysis}

The main objective of the present study is to determine the contact phenomena between the robot finger $\left(\right.$ Viton $^{\mathrm{R}}$ rubber) and a spherical object (ball) for a single asperity contact under loading conditions. The finite element analysis of the contact parameters is carried out by the ABAQUS software. The analysis is done under an axisymmetric condition. The assumption made in the study is that the robot fingers are in contact with the spherical object. A quarter sphere and a flat model are considered for the simulation study [25]. Figure 3 shows the finite element model used in the present study. The element type CAX4R is considered in the study. The boundary condition considered for the analysis is that the radial direction movements of the symmetric axis nodes are constrained, the bottom of the flat is fixed and the reference point (RP) is chosen at the centre of the sphere. The contact formulation has been made as a frictionless contact model.

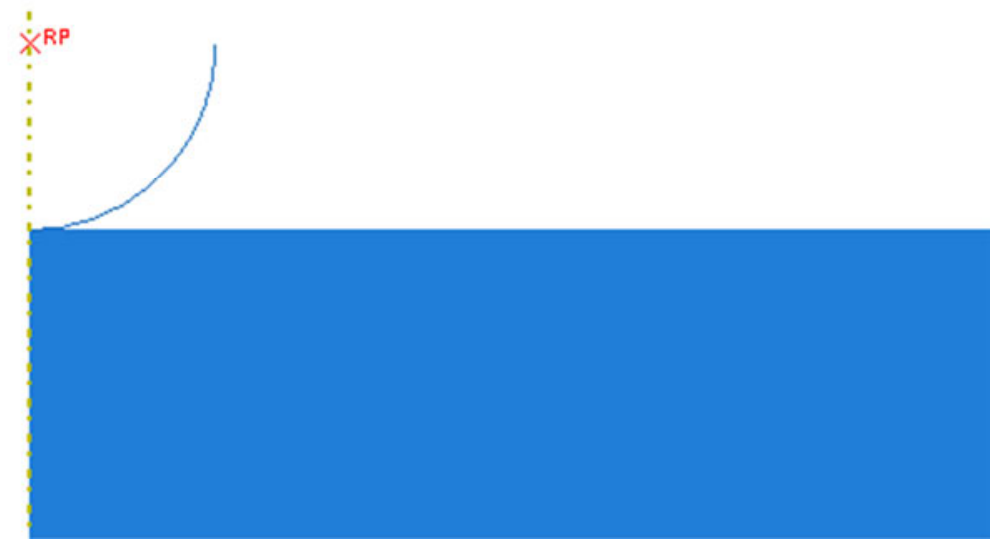

Fig. 3 Finite element model

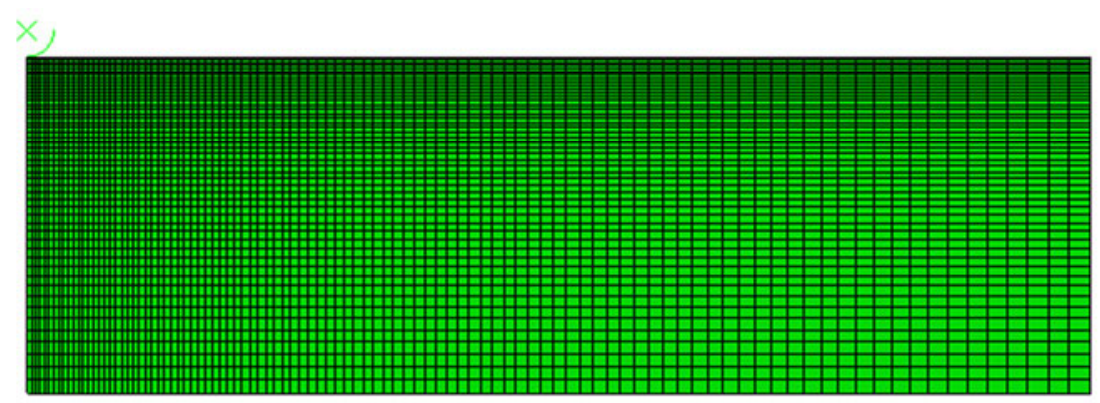

Fig. 4 Finite element meshed model

Figure 4 shows the finite element meshed model of a sphere and a flat model. The fine mesh is carried out in the top and the left edge of the flat plate. The sphere of $10 \mathrm{~mm}$ in diameter and a total number of 5150 elements is considered for the analysis. Various depths of indentation, i.e. 4,7 , and $9.5 \mathrm{~mm}$, for the loads of 50,100 , and $150 \mathrm{~N}$, respectively, are analysed. The values of loads have been chosen according to the forces chosen to measure the contact width. The displacement $(4,7,9.5 \mathrm{~mm})$ is given for the sphere that has penetrated the flat plate. The Von-Mises stress and contact pressure are estimated for the silicone and Viton ${ }^{\mathrm{R}}$ rubbers in contact with a steel sphere. 
D. Sivanesan, U. Natarajan, V.C. Sathish Gandhi
Experimental and Finite Element Studies of a Soft Robot Finger Material - a Contact Mechanics Approach

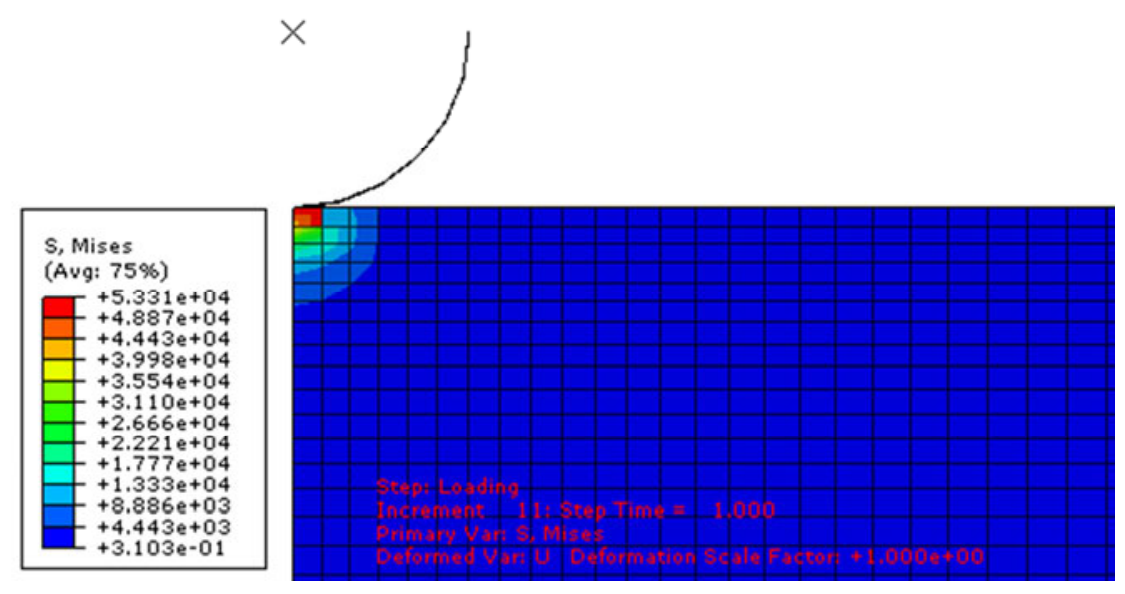

Fig. 5 (a) Von-Mises stress - 4mm displacement of the sphere

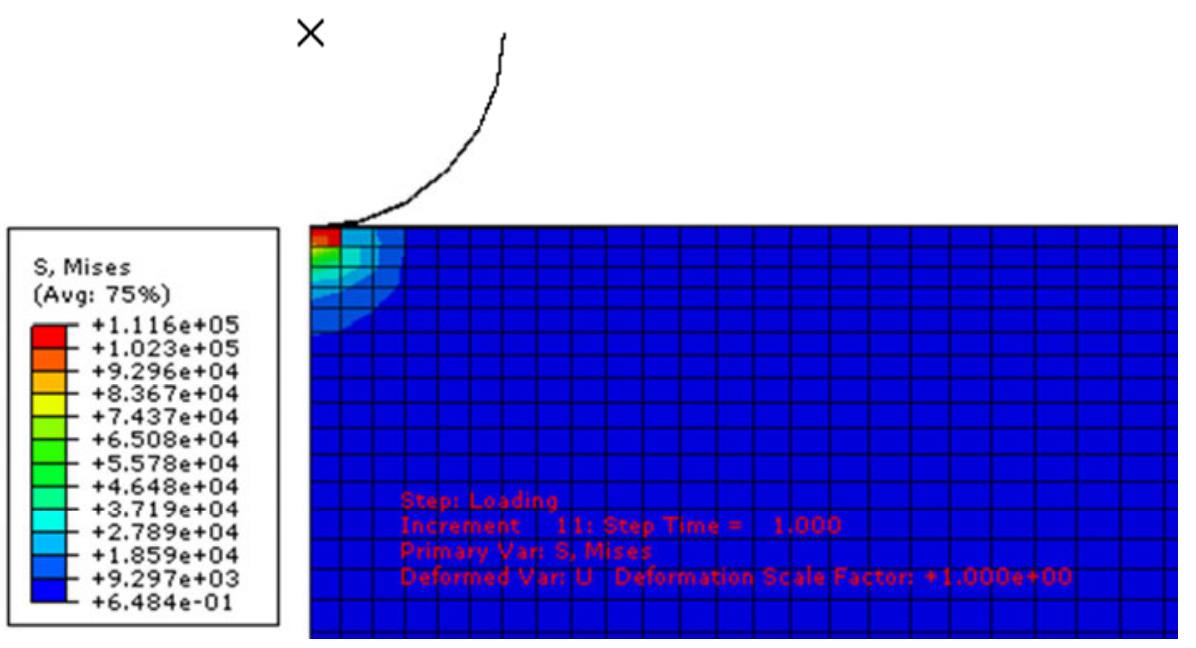

Fig. 5 (b) Von-Mises stress - 7mm displacement of the sphere

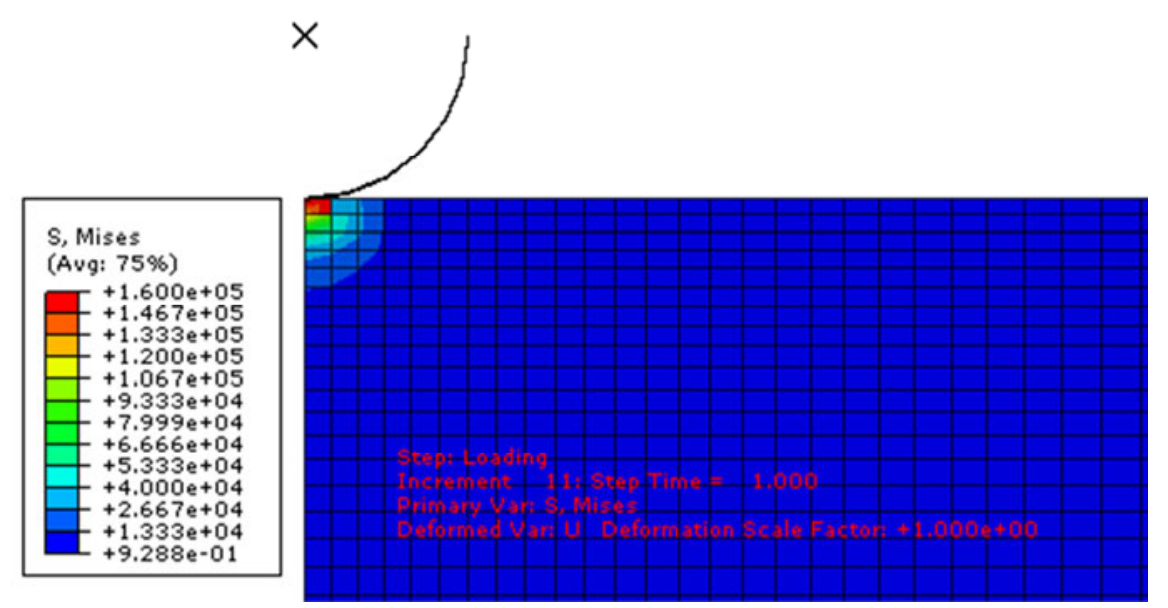

Fig. 5 (c) Von-Mises stress $-9.5 \mathrm{~mm}$ displacement of the sphere

For different depths of indentation, the Von-Mises stress developed in the flat plate (Viton $^{\mathrm{R}}$ rubber) is shown in Fig. 5(a), (b), and (c). A point where the flat plate comes in contact with the sphere exhibits maximum stress. The increment in the stress value is noted as the depth of indentation increases. 




Fig. 6 (a) Contact pressure - 4mm displacement of the sphere

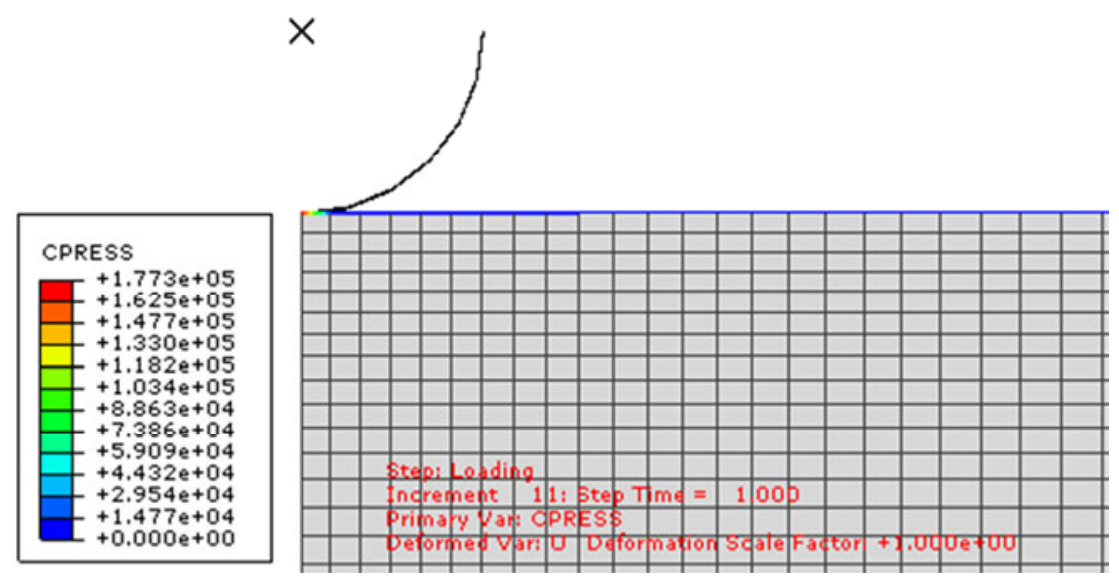

Fig. 6 (b) Contact pressure $-7 \mathrm{~mm}$ displacement of the sphere

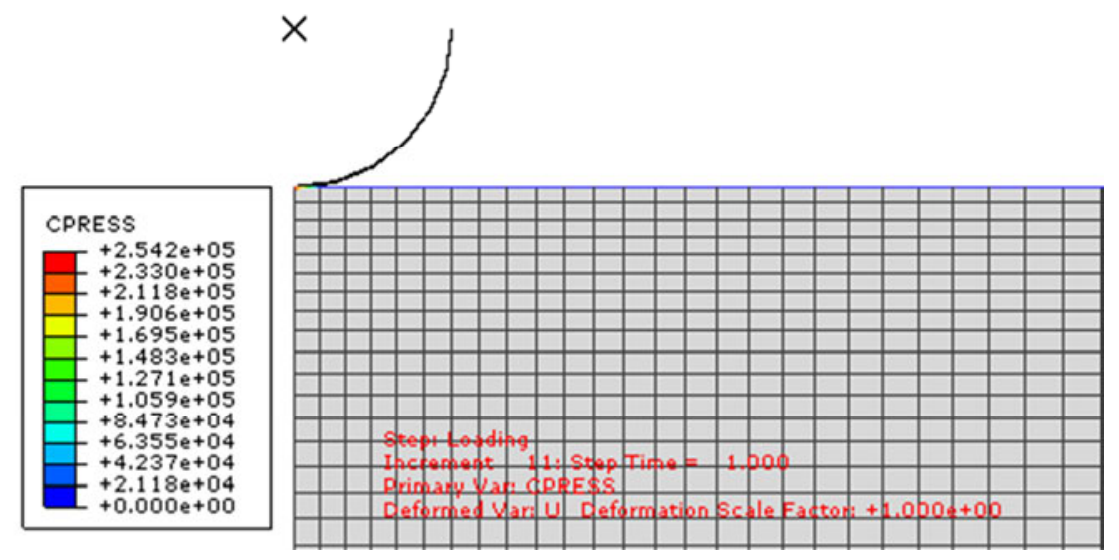

Fig. 6 (c) Contact pressure $-9.5 \mathrm{~mm}$ displacement of the sphere

For different depths of indentation, the development of contact pressure between the sphere and the flat plate (Viton ${ }^{\mathrm{R}}$ rubber) is shown in Fig. 6(a), (b), and (c). A point where the flat plate comes in contact with the sphere exhibits maximum contact pressure. The increment in the contact pressure value is noted with an increase in the depth of indentation.

\section{Results and discussion}

The values of tensile strength, hardness, elongation (at break), Young's modulus, VonMises stress, and contact pressure and width are determined by simulation and experimental studies. This study is carried out to identify the suitability of the material for the robot finger with the capability of soft handling and also of withstanding higher temperatures. 
The contact width for different loads has been estimated in the simulation study. The results are shown in Table 6. It is observed that the percent deviation of the contact width in the simulation study of displacements of sphere of 4,7 , and $9.5 \mathrm{~mm}$ and that in the experimental study is less than $6.5 \%$. Hence, it is shown that the simulation results are within the acceptable values.

Table 6 Simulation and experimental results - Contact width

\begin{tabular}{|c|c|c|c|c|}
\hline \multirow{2}{*}{$\begin{array}{c}\text { Sl. } \\
\text { No. }\end{array}$} & \multirow{2}{*}{ Force of grasping / N } & \multicolumn{2}{|c|}{ Contact width / mm } & \multirow{2}{*}{ \% Deviation } \\
\cline { 3 - 4 } & & Experimental & Simulation & \\
\hline 1 & 50 & 3.89 & 4.15 & 6.26 \\
\hline 2 & 100 & 4.59 & 4.70 & 2.34 \\
\hline 3 & 150 & 5.25 & 5.45 & 3.67 \\
\hline
\end{tabular}

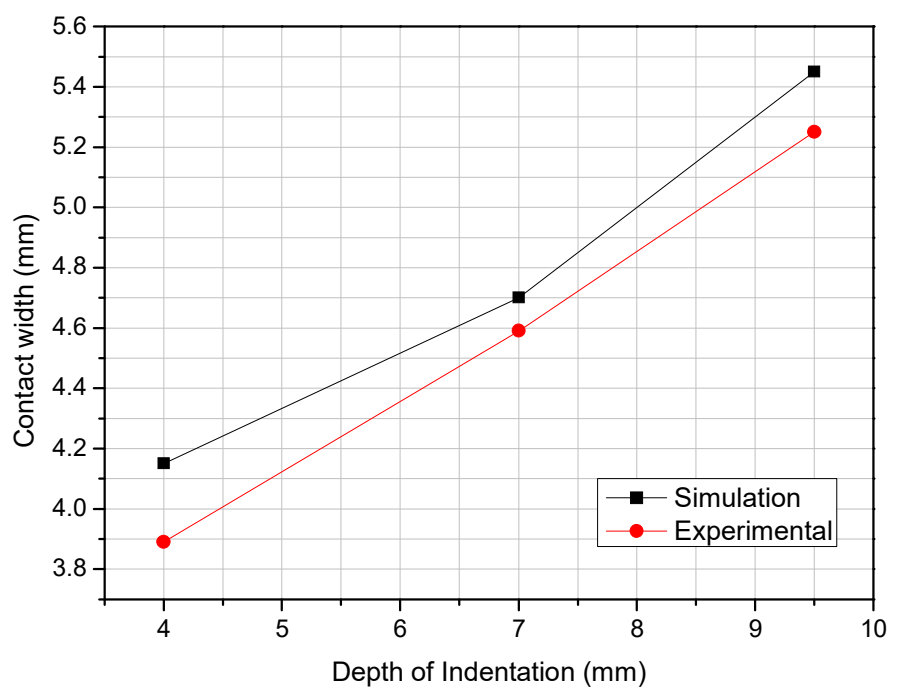

Fig. 7 Depth of indentation vs contact width

Figure 7 shows the relationship between the depth of indentation and the contact width in the experimental and the simulation study. It is observed that the depth of indentation gradually increased the contact width (the contact area between the finger and the object being held by the robot). It is shown that the Viton ${ }^{\mathrm{R}}$ rubber has the capability of increasing the force and of transmitting the required force to the finger to hold the object. In nature, this material shows a nonlinear behaviour if the load is continuously applied to the material. Hence, the material shows a non-linear behaviour.

Table 7 Results of experimental work

\begin{tabular}{|c|l|c|c|}
\hline S. No. & \multicolumn{1}{|c|}{ Properties } & $\begin{array}{c}\text { Silicone } \\
\text { rubber }\end{array}$ & $\begin{array}{c}\text { Viton }^{\mathrm{R}} \\
\text { rubber }\end{array}$ \\
\hline 1 & Hardness & 65 & 75 \\
\hline 2 & Tensile strength $/ \mathrm{N} / \mathrm{mm}^{2}$ & 5.20 & 8.46 \\
\hline 3 & Elongation (at break), \% & 162.28 & 203.68 \\
\hline
\end{tabular}

Better mechanical properties are observed in the Viton $^{\mathrm{R}}$ rubber than in the silicone rubber, as shown in Table 7. Thus, the Viton ${ }^{\mathrm{R}}$ rubber is considered for further studies. Then, simulation is done to study the Von-Mises stress, contact pressure, and contact width. 
Table 8 Results - simulation

\begin{tabular}{|c|c|c|c|}
\hline S. No. & Depth of indentation / $\mathrm{mm}$ & Contact pressure $/ \mathrm{N} / \mathrm{m}^{2}$ & Von-Mises stress / N/m ${ }^{2}$ \\
\hline 1 & 4 & $8.473 \times 10^{4}$ & $5.331 \times 10^{4}$ \\
\hline 2 & 7 & $1.773 \times 10^{5}$ & $1.116 \times 10^{5}$ \\
\hline 3 & 9.5 & $2.542 \times 10^{5}$ & $1.600 \times 10^{5}$ \\
\hline
\end{tabular}

For the model of the robot finger holding an object with different depths of indentation, the values of the Von-Mises stress and contact pressure are given in Table 8. The increments in the values of the Von-Mises stress and contact pressure are observed when there is an increment in the indentation depth. The validation of the developed model for the object and the robot finger is done using the results obtained by simulation.

\section{Conclusion}

The present study has been carried out in order to select a soft material for the robot finger, which would be capable of withstanding high temperatures. The materials considered in the present study are the $\operatorname{Viton}^{\mathrm{R}}$ and the silicone rubber. The experimental study is carried out for both materials. The properties considered for estimation are percent elongation (at break), tensile strength, contact width, and hardness. The finite element analysis software "ABAQUS" is used for the simulation study. The contact parameters, i.e. Von-Mises stress, contact width and contact pressure between the sphere and the flat plate, are estimated in the simulation using different depths of indentation. The experimental results show that the Viton ${ }^{\mathrm{R}}$ rubber is better for the design of the robot finger because this material is soft in nature and can withstand higher temperatures than the silicone rubber. Thus, in order to avoid damage while handling objects, the Viton $^{\mathrm{R}}$ rubber will be used for the robot finger as it enables the gentle handling of the object.

\section{REFERENCES}

[1] Antonio Bicchi and Vijaykumar: Robotic Grasping and contact: A review. Proceedings of the IEEE International conference on Robotics and Automation (2000).

[2] Salisbury J. K: Kinematics and force analysis of articulated hands. PhD Thesis, Stanford University (1982).

[3] Hertz. H: On the contact of elastic solids. J. Reine undund angewandel Mathematik, 92, 156-171(1982).

[4] Pawluk, D and Howe R: Dynamic lumped element response of the Human finger pad. Journal of Biomechanics, 121, 178-183 (1999). https://doi.org/10.1115/1.2835100

[5] Asada, H: Studies on Prehension and handling by robot hands with elastic fingers. Ph.D Dissertation, Kyoto University (1979).

[6] Jacobsen, S. C, Wood, J. E., Biggers, K. B: The UTAH - MIT Dexterous Hand, Mechanical and Industrial Engineering", University of Utah, Salt Lake City, Utah-84112 (2006).

[7] Sinha, P. R., Abel, J. M: A contact stress model for multi fingered Grasps for rough Objects. IEEE Trans on Robotics and Automation, 8 (1), 7-22 (1992). https://doi.org/10.1109/70.127235

[8] Wang, Y.T, Kumar, V., Abel, J. Dynamics of Rigid body with multiple frictional Contacts. Proceedings of IEEE Intl conf of Robotics and Automation, Nice, France (1992).

[9] Serina, E., Mote, C., Rempel, D.: A structural model of the forced compression of the fingertip pulp. Journal of Bio mechanics, 31, 639-646 (1998). https://doi.org/10.1016/S0021-9290(98)00067-0

[10] Berselli, G., and Vassura, G.: Nonlinear Modeling and Experimental Evaluation of Fluid filled Soft pads for robotic hands. $9^{\text {th }}$ Youth Symposium on experimental Solid Mechanics, Trieste, Italy, (2010).

[11] Xydis, N. and Koa, I.: Modeling of contact Mechanics and Friction Limit surface for soft finger with experimental Results", International Journal of Robotic Research, 18(9), 941- 950 (1999). https://doi.org/10.1177/02783649922066673 
[12] Jiyong Hu, Ding Xin, Rubin Wang: Dependence of tactile sensation on deformations within Soft tissues of fingertip", World Journal of Modeling and Simulation, 3(1), 73-78(2007).

[13] Fanny Ficuciello: Modeling and Control for Soft Finger Manipulation and Human-Robot Interaction. Research Article, T esi di Dottorato di Ricerca in Ingegneria Informatica ed Automatica (2012).

[14] Subramaniam, P. and Marappan, R.: Semi Cylindrical Fluid Filled Hyper elastic Finger Model for Soft Contact. International Journal of Engineering and Technology, 5 (6), 1131-1137(2013).

[15] Elango, N and Faudzi, A. A. M.: A review article: investigations on soft materials for soft robot manipulations. The International Journal of Advanced Manufacturing Technology, 80(5), 1027-1037 (2015). https://doi.org/10.1007/s00170-015-7085-3

[16] Blanes, C., Mellado, M., Beltran, P.: Novel Additive Manufacturing Pneumatic Actuators and Mechanisms for Food Handling Grippers. Actuators , 3, 205-225(2014). https://doi.org/10.3390/act3030205

[17] Elango, $\mathrm{N}$ and Marappan, R: Analysis on the fundamental deformation effect of a robot soft finger and its contact width during power grasping. The International Journal of Advanced Manufacturing Technology, 52 (5-8), 797-804(2011). https://doi.org/10.1007/s00170-010-2747-7

[18] She,Y., Li, C., Cleary, J., Su, H.J.: Design and Fabrication of a Soft Robotic Hand With Embedded Actuators and Sensors. J. Mechanisms Robotics, 7(2), 021007-021015 (2015). https://doi.org/10.1115/1.4029497

[19] Cabibihan, J. J., Chauhan, S.S., Suresh, S.: Effects of the Artificial Skin's Thickness on the Subsurface Pressure Profiles of Flat, Curved, and Braille Surfaces" IEEE Sensors Journal, 14(7), 2118 - 2128 (2014). https://doi.org/10.1109/JSEN.2013.2295296

[20] Elango, N, Faudzi, A. A. M., Hassan, A and Rusydi, M. R. M : Experimental Investigations of a Skin-like Material and the Computation of its Material Properties", International Journal of Precision Engineering and Manufacturing, 15(9), 1909-1914 (2014). https://doi.org/10.1007/s12541-014-0545-0

[21] Elango, N, Faudzi, A. A. M., Rusydi, M. R. M and Nordin, I. N. A. M. : Determination of Non linear Material Constants of RTV Silicone Applied to a Soft Actuator for Robotic Applications. Key Engineering Materials, 95, pp. 1099-1104 (2013). https://doi.org/10.4028/www.scientific.net/KEM.594-595.1099

[22] Tiezzi P., Lotti F. and Vassura G.: Polyurethane gel pulps for robotic fingers. Proceedings of IEEE International Conference on Advanced Robotics, ICAR 03, Italy, 1-7 (2003).

[23] Gent, A. N.: On the relation between indentation hardness and Young's modulus. Rubber Chemistry and Technology, 34 (4), 896-906 (1958). https://doi.org/10.5254/1.3542351

[24] Mix, A. W. and Giacomin, A. J.: Standardized polymer durometry. Journal of Testing and Evaluation, 39 (4), 1-10 (2011).

[25] Sathish Gandhi, V. C., Ramesh, S., Kumaravelan, R., Thanmanaselvi, M. : Contact analysis of spherical ball and a deformable flat model with the effect of tangent modulus. Structural Engineering and Mechanics, An International Journal, 44(1), 61-72 (2012). https://doi.org/10.12989/sem.2012.44.1.061

Submitted: $\quad 22.9 .2017$

Accepted: $\quad$ 08.5.2018
Desingh Sivanesan

Department of Mechanical Engineering, University College of Engineering, Ariyalur, Tamilnadu, India Dr. Uthirapathy Natarajan Department of Mechanical Engineering, Alagappa Chettiar College of Engineering and Technology, Karaikudi, Tamilnadu, India.

Veeramalai Chinnasamy Sathish Gandhi Department of Mechanical Engineering, University College of Engineering, Nagercoil, Konam, Tamilnadu, India. 